\title{
Using Python to Study Rotational Velocity Distributions of Hot Stars
}

\author{
Gustavo Bragança $^{\S *}$, Simone Daflon ${ }^{\S}$, Katia Cunha**, Thomas Bensby ${ }^{\ddagger}$, Sally Oey ${ }^{\natural}$, Gregory Walth
}

\begin{abstract}
Stars are fundamental pieces that compose our Universe. By studying them we can better comprehend the environment in which we live. In this work, we have studied a sample of 350 nearby $O$ and $B$ stars and have characterized them in aspects of their multiplicity, temperature, spectral classifications, and projected rotational velocity.

Python is a robust language with a steep learning curve, i.e. one can make rapid progress with it. In this proceeding, we will present how we used Python in our research.
\end{abstract}

Index Terms-Astronomy, Stars, Galactic Disk

\section{Introduction}

The study of $\mathrm{O}$ and $\mathrm{B}$ stars is an important key to understanding how star formation occurs. When these stars are born, they have the greatest mass, temperature and rotation. Their mass can go from 2.5 up to 120 times the Solar mass, their temperatures ranging from $11,000 \mathrm{~K}$ up to $60,000 \mathrm{~K}$, and rotation up to 400 $\mathrm{km} / \mathrm{s}$.

By definition, a star is born when it starts synthesizing Hydrogen into Helium through nuclear fusion. The star performs this nucleosynthesis during some $90 \%$ of their life. When stars are at this stage, they are called dwarfs. Most of the studied stars on this work are dwarfs. Due to their young age, dwarf stars have not lost too much of their mass, and so, the majority of their stellar properties are kept unchanged. This helps us understand how these stars formed.

Stars are born inside molecular clouds and, usually, a molecular cloud can generate several stars. After their formation, these stars compose a stellar association, that, in its infancy, is still gravitationally bounded. With their unchanged properties, it is possible to trace the membership of these stars and then verify if some stars are from the same association.

The Python programming language is very powerful, robust, clean and easy to learn. The scripting nature allows the programmer to have a dynamic workflow and not lose too much time with debugging and compiling. With a set of packages, like Scipy,

* Corresponding author: ga.braganca@gmail.com

$\S$ Observatório Nacional, Brazil

** Observatório Nacional, Brazil; National Optical Astronomy Observatory, University of Arizona, U. S. A.

\$ Lund Observatory, Sweden

II University of Michigan, U. S. A.

II Steward Observatory, U. S. A.

Copyright $\odot 2013$ Gustavo Bragança et al. This is an open-access article distributed under the terms of the Creative Commons Attribution License, which permits unrestricted use, distribution, and reproduction in any medium, provided the original author and source are credited.
Numpy and Matplotlib, Python becomes very suited for scientific research. On the last years, it has been widely adopted in the Astronomic community and several astronomical packages are being translated to Python or just recently being created. All of these motivated us to use Python in our research.

In this proceedings, we relate how we used Python in our research. A more profound scientific analysis can be found at [Brag12].

\section{Research development \\ Sample Characterization}

The observed sample of stars is displayed in Figure 1 in terms of their Galactic longitude and heliocentric distance projected onto the Galactic plane. The stars in the sample are all nearby $(\sim 80 \%$ are within $700 \mathrm{pc}$ ) and relatively bright $(V \sim 5-10)$.

We used Python allied to the Matplotlib package to construct the plot presented in Figure 1 and all plots of this work. The code for this plot is:

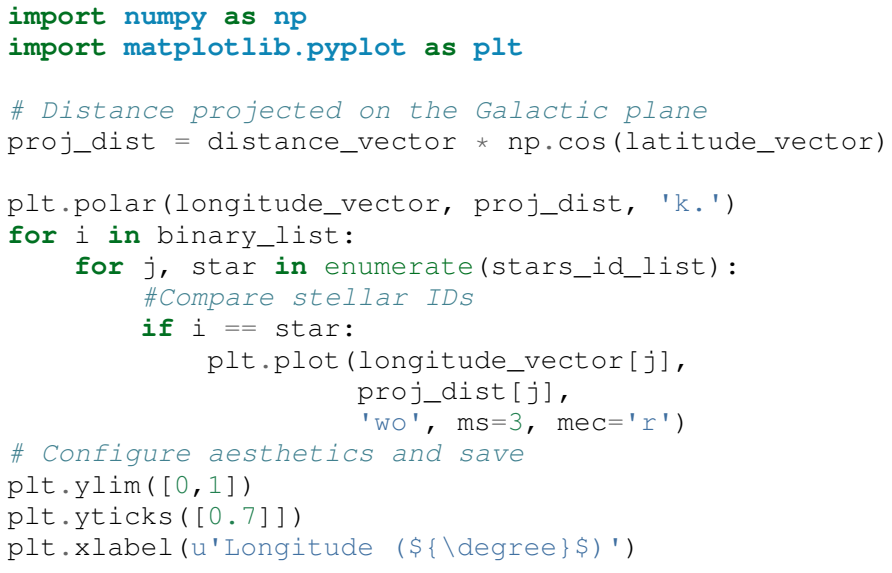

As we have said before, stars usually are born in groups. Thus, a great majority of them are binaries or belong to multiple systems. For a spectroscopic study, as was this, the only problem occurs when the spectrum of one observation has two or more objects. The identification of these objects was done on a visual inspection and with support of the works of [Lefe09] and [Egle08]. Since the study of these stars was outside the scope of our project, we discarded them. These objects are represented in Figure 1 as red circles.

Our sample is composed of high-resolution spectroscopic observations with wavelength coverage from 3350 up to 9500 Angstrons. Sample spectra are shown in Figure 2 in the spectral 


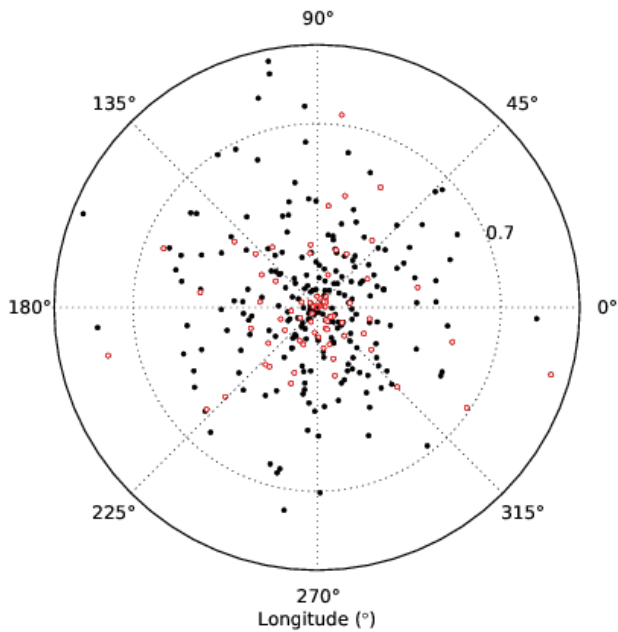

Fig. 1: Polar plot showing the positions of the sample stars projected onto the Galactic plane. The plot is centered on the Sun. The open red circles are spectroscopic binaries/multiple systems identified in our sample.

region between 4625 and 4665 Angstrom, which contains spectral lines of $\mathrm{C}, \mathrm{N}, \mathrm{O}$, and $\mathrm{Si}$. The code to plot this Figure is:

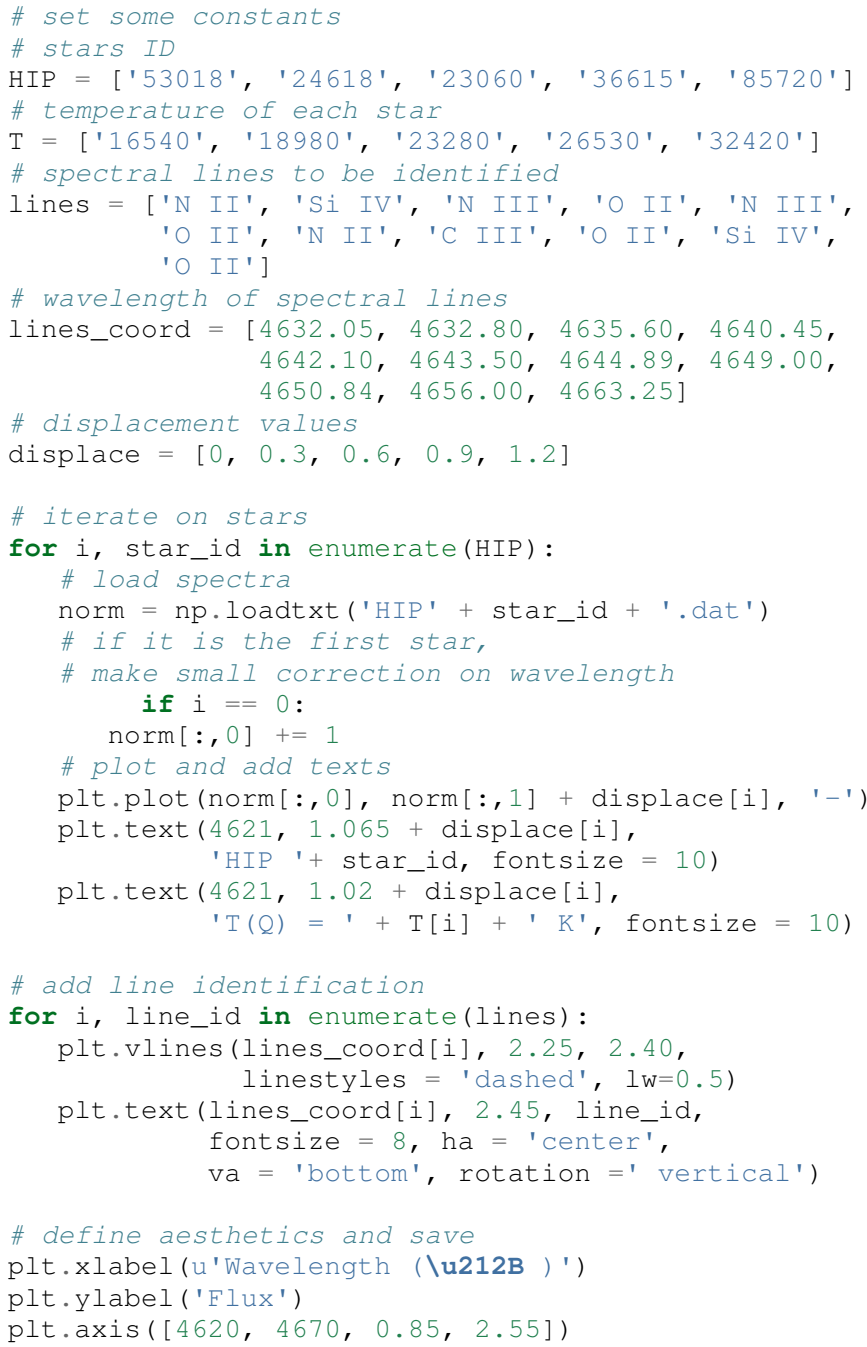

To analyze the spectra images we have used IRAF (Image and Reduction Analysis Facility), which is a suite of softwares to

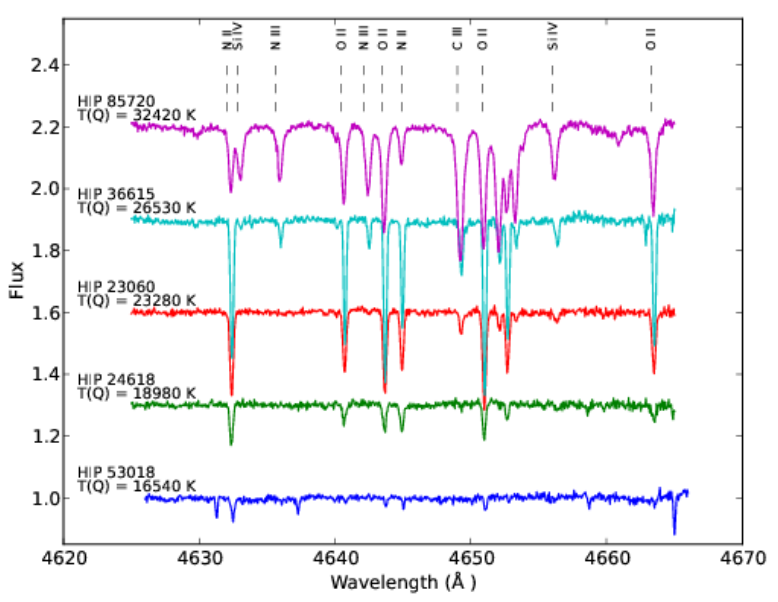

Fig. 2: Example spectra of five sample stars in the region 46254665 Angstrom. Some spectral lines are identified. The spectra were arbitrarily displaced in intensity for better viewing.

handle astronomic images developed by the $\mathrm{NOAO}^{1}$. We had to do several tasks on our spectra (e.g. slice them at a certain wavelength and normalization) to prepare our sample for further analysis. Some of these tasks had to be done manually and on a one-byone basis, but some others were automated. The automation could have been done through IRAF scrips, but fortunately, the STSCI ${ }^{2}$ has developed a Python wrapper for IRAF called PyRAF. For example, we show how we used the IRAF task SCOPY to cut images from a list using pyRAF:

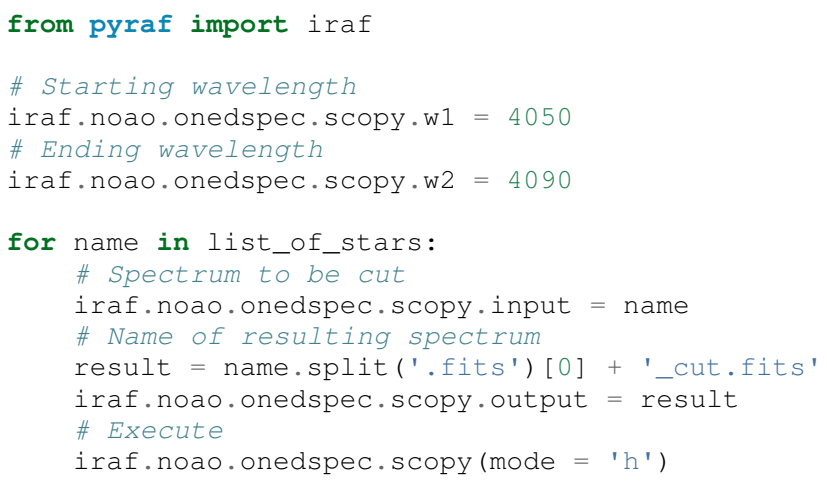

We also have performed a spectral classification on the stars and, since this was not done using Python, more information can be obtained from the original paper.

We have obtained effective temperature (Teff) from a calibration presented in [Mass89] that uses the photometric reddeningfree parameter index $Q$ ([John58]).

A histogram showing the distribution of effective temperatures for OB stars with available photometry is shown in Figure 3. The effective temperatures of the target sample peak around $17,000 \mathrm{~K}$, with most stars being cooler than $28,000 \mathrm{~K}$.

\section{Projected rotational velocities}

We have obtained projected rotational velocities $(v \sin i)$ for 266 stars of our sample (after rejecting spectroscopic binaries/multiple systems) using measurements of full width at half maximum of

1. National Optical Astronomy Observatory

2. Space Telescope Science Institute 


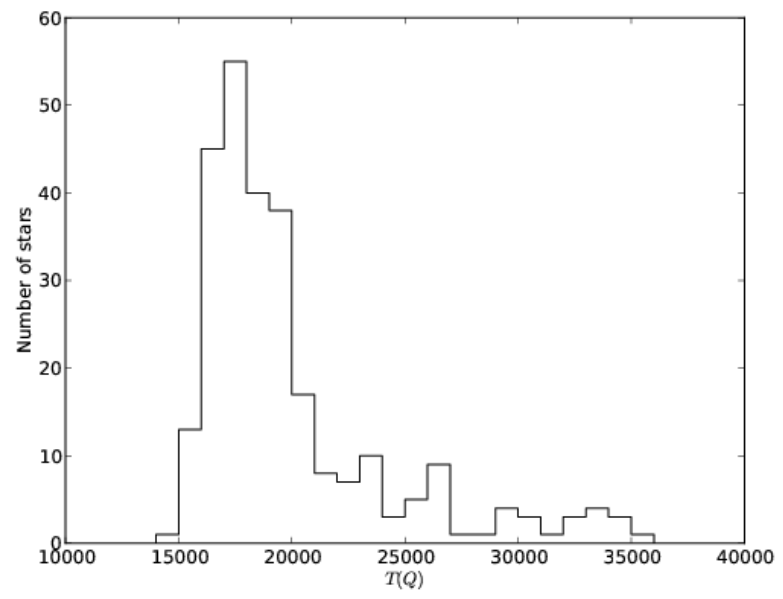

Fig. 3: Histogram showing the distribution of effective temperatures for the studied sample.

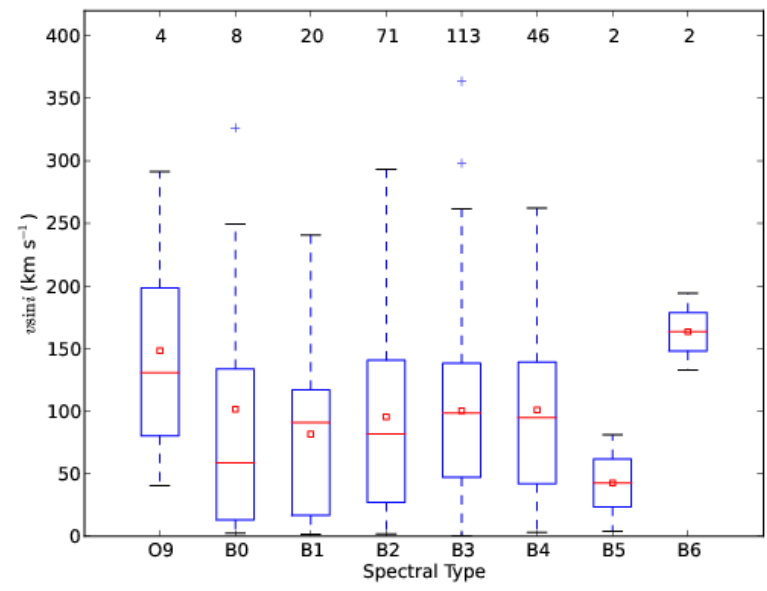

Fig. 4: Box plot for the studied stars in terms of the spectral type. The average $v \sin i$ for the stars in each spectral type bin is roughly constant, even considering the least populated bins.

He I lines and interpolation in a synthetic grid from [Dafl07]. We did not use Python to obtain $v \sin i$, so, for more information, we suggest the reader to look in the original paper. However, to analyze the stars $v \sin i$ we used Python, especially the matplotlib package for visualization analysis and the Scipy.stats package for statistics analysis.

The boxplot is a great plot to compare several distributions side by side. In this work, we used a boxplot to analyze the $v \sin i$ for each spectral type subset, as can be seen in Figure 4. The average $v \sin i$ for the stars in each spectral type bin is roughly constant, even considering the least populated bins. The code used to plot it was:

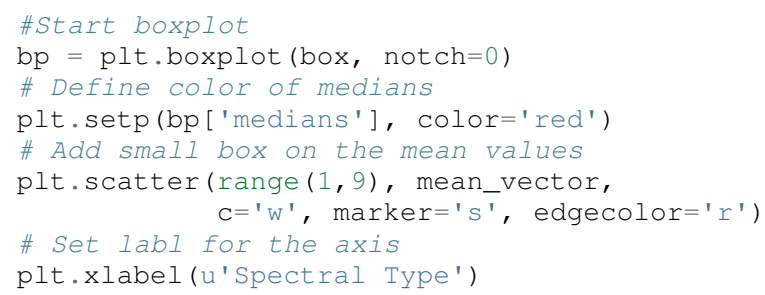

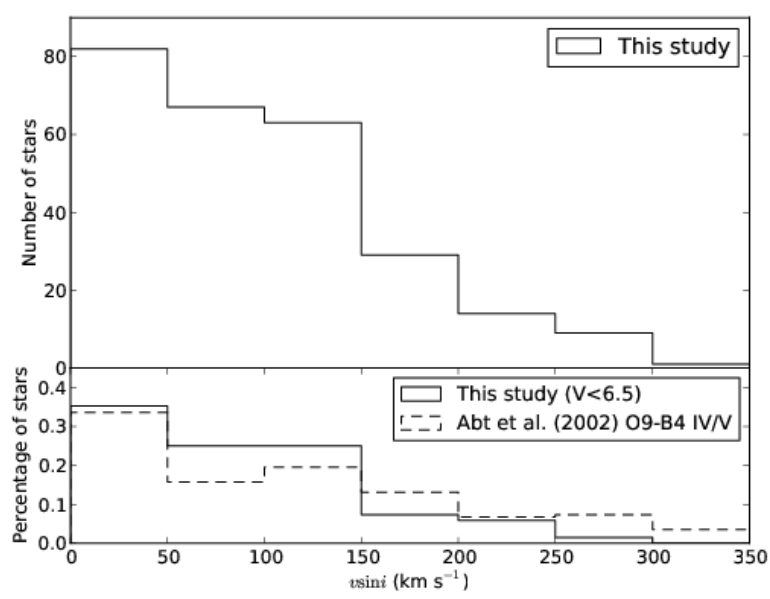

Fig. 5: Histogram of $v \sin i$ distribution of our sample on the top panel. The bottom panel compares the normalized distribution of a subsample of stars in our sample with a magnitude cut in $V=6.5$ and a sample with 312 field stars (spectral types O9-B4 IV/V) culled from [Abt02].

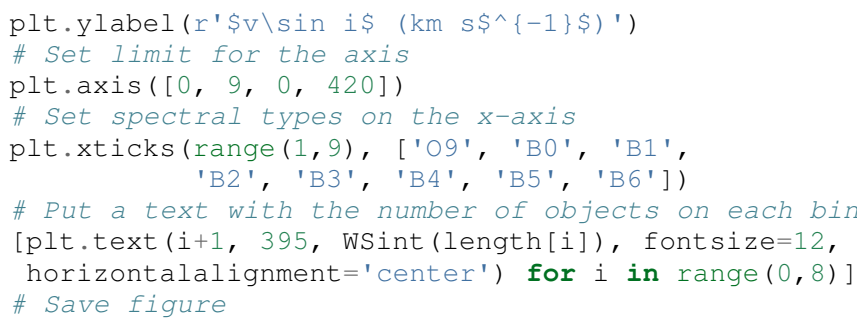

And the distribution of $v \sin i$ for the stars of our sample is presented on Figure 5. The distribution has a modest peak at low $v \sin i(\sim 0-50 \mathrm{~km} / \mathrm{s})$ but it is overall flat (a broad distribution) for $v \sin i$ roughly between 0 and $150 \mathrm{~km} / \mathrm{s}$; the number of stars drops for higher values of $v \sin i$. [Abt02] provide the cornerstone work of the distributions of projected rotational velocities of the so-called field OB stars. To compare our sample with Abt's, we subselected our sample on magnitude and Abt's sample on spectral type. Both distributions are shown on the bottom panel of Figure 5. The code used to build this plot follows:

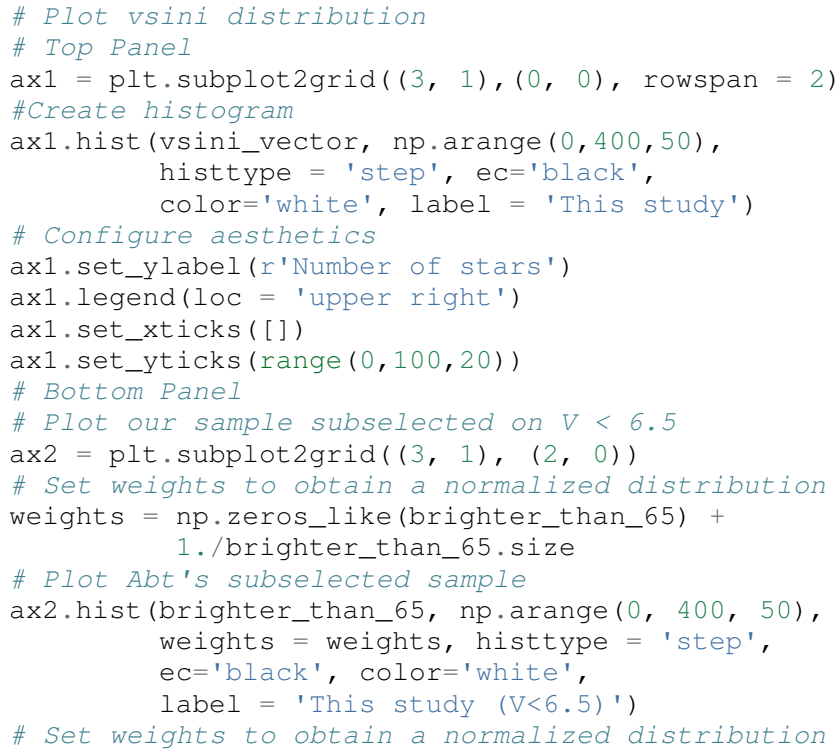



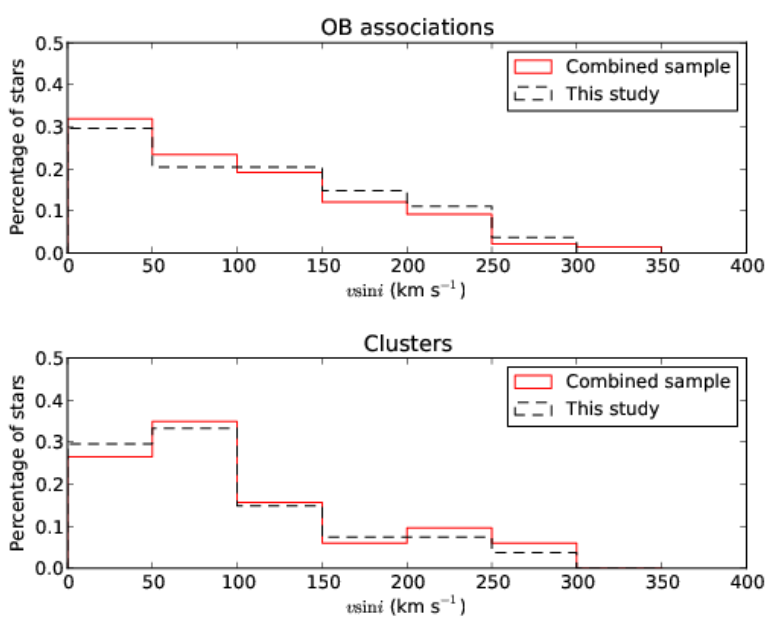

Fig. 6: Distribution of $v \sin i$ for the studied samples of $O B$ association (top panel) and cluster members (lower panel) are shown as red dashed line histograms. The black solid line histograms represent the combined sample: stars in this study plus 143 star members of clusters and associations from [Dafl07]. Both studies use the same methodology to derive $v \sin i$.

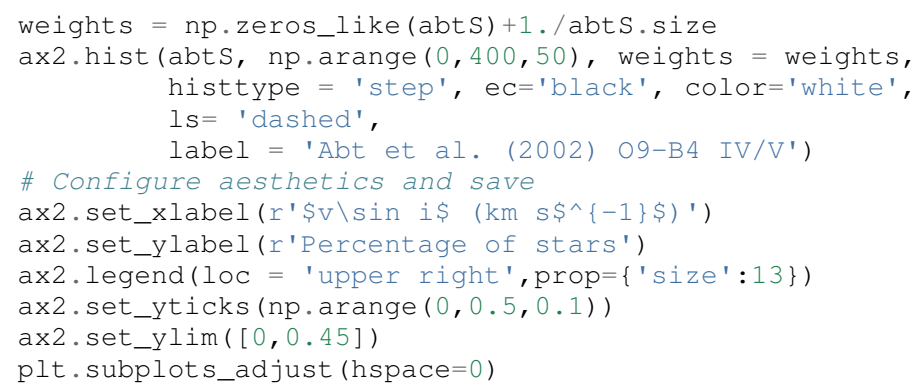

There is evidence that there are real differences between the $v \sin i$ distributions of cluster members when compared to field ([Wolf07], [Huan08]); there are fewer slow rotators in the clusters when compared to the field or the stars in clusters tend to rotate faster. Using literature results ([Hump84], [Brow94], [Zeeu99], [Robi99], [Merm03], [Tetz11]), we separated our sample into four different categories according to the star's membership: field, cluster, association and runaway. We have merged our sample with that of [Dafl07] in which their results were obtained using the same methodology as ours. We present in Figure 6 the distributions of stars belonging to clusters and from associations.

We have used the Kolmogorov-Smirnov (KS) statistics to test the null hypothesis that membership subsamples are drawn from the same population. For this we used the ks_2samp task available on the scipy.stats package. The resulting values are available in Table 1 . Note that, any differences between the distributions of clusters and associations in this study are not very clear and may not be statistically significant; larger studies are needed. Also, the runaway subsample seems to be more associated with the dense cluster environments, as expected from a dynamical ejection scenario.

\section{Conclusions}

We have investigated a sample of 350 OB stars from the nearby Galactic disk. Our focus was to realize a first characterization of this sample. We obtained effective temperature using a photometric calibration and determined that the temperature distribution

\begin{tabular}{lllll}
\hline & Field & Association & Cluster & Runaway \\
Field & -- & $92 \%$ & $88 \%$ & $18 \%$ \\
Association & $92 \%$ & -- & $50 \%$ & $40 \%$ \\
Cluster & $88 \%$ & $50 \%$ & - & $71 \%$ \\
Runaway & $18 \%$ & $40 \%$ & $71 \%$ & -- \\
\hline
\end{tabular}

TABLE 1: Resulting values for the KS test for the membership groups.

peaks around $17,000 \mathrm{~K}$, with most stars being cooler than 28,000 K.

We calculated the projected rotational velocities using the full width at half measure of He I lines and found that the distribution has a modest peak at low $v \sin i(\sim 0-50 \mathrm{~km} / \mathrm{s})$ but it is overall flat (a broad distribution) for $v \sin i$ roughly between 0 and $150 \mathrm{~km} / \mathrm{s}$; the number of stars drops for higher values of $v \sin i$.

We subselected our sample on a membership basis and, when the OB association and cluster populations are compared with the field sample, it is found that the latter has a larger fraction of slowest rotators, as previously shown by other works. In fact, there seems to be a gradation from cluster to $\mathrm{OB}$ association to field in $v \sin i$ distribution.

We have constantly used Python in the development of this work. In our view, the advantages of Python are the facility of learning, the robust packages for science and data analysis, a plot package that renders beautiful plots in a fast and easy way, and the increase of packages for the astronomic community.

\section{Acknowledgments}

We warmly thank Marcelo Borges, Catherine Garmany, John Glaspey, and Joel Lamb for fruitful discussion that greatly improved the original work. G.A.B. thanks the hospitality of University of Michigan and of NOAO on his visit, Leonardo Uieda and Katy Huff for their help in this proceedings and also thanks all Python developers for their great work. G.A.B. also acknowledges Conselho Nacional de Desenvolvimento Científico e Tecnológico (CNPq-Brazil) and Coordenação de Aperfeiçoamento de Pessoas de Nível Superior (CAPES - Brazil) for his fellowship. T.B. was funded by grant No. 621-2009-3911 from the Swedish Research Council (VR). M.S.O. and T.B. were supported in part by NSFAST0448900. M.S.O. warmly thanks NOAO for the hospitality of a sabbatical visit. K.C. acknowledges funding from NSF grant AST-907873. This research has made use of the SIMBAD database, operated at CDS, Strasbourg, France.

\section{RefERENCES}

[Abt02] Abt, H. A., Levato, H., Grosso, M., Astrophysical Journal, 573: 359, 2002

[Brag12] Braganca, G. A, et al., Astronomical Journal, 144:130, 2012.

[Brow94] Brown, A. G. A., de Geus, E. J., de Zeeuw, P. T., Astronomy \& Astrophysics, 289: 101, 1994

[Dafl07] Daflon, S., Cunha, K., de Araujo, F. S. W., \& Przybilla, N., Astronomical Journal, 134:1570, 2007

[Egle08] Eggleton, P. P., \& Tokovinin, A. A., M.N.R.A.S., 389:869, 2008

[John58] Johnson, H. L., Lowell Obs. Bull., 4:37, 1958

[Huan08] Huang, W., \& Gies, D. R., Astronomical Journal, 683: 1045, 2008

[Hump84] Humphreys, R. M., McElroy, D. B., Astrophysical Journal, 284:565, 1984

[Lefe09] Lefevre, L., Marchenko, S. V., Moffat, A. F. J., Acker, A., Astronomy \& Astrophysics, 507:1141, 2009

[Mass89] Massey, P., Silkey, M., Garmany, C. D., Degioia-Eastwood, K., Astronomical Journal, 97:107, 1989, 
[Merm03] Mermilliod, J.-C., Paunzen, E., Astronomy \& Astrophysics, 410:51, 2003

[Robi99] Robichon, N., Arenou, F., Mermilliod, J.-C., Turon, C., Astronomy \& Astrophysics, 345:471, 1999

[Tetz11] Tetzlaff, N., Neuhäuser, R., Hohle, M. M., M.N.R.A.S., 410:190, 2011

[Wolf07] Wolff, S. C., Strom, S. E., Dror, D., \& Venn, K., Astronomical Journal, 133:1092, 2007

[Zeeu99] de Zeeuw, P. T., Hoogerwerf, R., de Bruijne, J. H. J., Brown, A. G. A., Blaauw, A., Astronomical Journal, 117:354, 1999 